\title{
TRA SFRUTTAMENTO E PROTESTA: I MIGRANTI E IL CAPORALATO AGRICOLO IN ITALIA MERIDIONALE
}

\author{
Between exploitation and protest: migrants and the agricultural \\ gangmaster system in Southern Italy
}

Angelo Scotto ${ }^{1}$

\begin{abstract}
Riassunto. Questo articolo descrive le caratteristiche e I'origine del caporalato (una forma di mediazione illecita all'assunzione lavorativa, in inglese "gangmaster system") nell'Italia meridionale, come è cambiato quando i migranti sono diventati la principale manodopera in agricoltura, il modo in cui porta allo sfruttamento dei lavoratori, e la reazione dei lavoratori stranieri contro questo sistema. Presentiamo due esempi di come i lavoratori hanno affrontato il caporalato in Puglia: nel primo caso, la provincia di Foggia, i migranti non sono stati in grado di organizzarsi per protestare contro i caporali; nel secondo caso, Nardò, hanno dato il via a uno sciopero che ha dato visibilità nazionale a questo fenomeno.
\end{abstract}

Parole chiave: caporalato, Italia meridionale, lavoratori stagionali stranieri, mobilitazione.

\begin{abstract}
This article describes the features and the origin of the gangmaster system (a form of illicit mediation to labour contracting, also called 'caporalato') in Southern Italy, how it has changed when migrant became the main workforce in agriculture, the way it leads to the exploitation of workers, and the reaction of foreign workers against this system. We provide two examples of how workers dealt with caporalato in Puglia: in the first case, the province of Foggia, migrants were not able to organize to protest against the caporals; in the second case, Nardò, they started a strike that gave this phenomenon national visibility.
\end{abstract}

Keywords: gangmaster system, Southern Italy, migrant seasonal workers, mobilization.

\footnotetext{
1 Dipartimento di studi politici e sociali, Università di Pavia. Pavia, Italia.
} 


\section{Introduzione}

Obiettivo di questo articolo è osservare e descrivere se e come gli immigrati in Italia che si trovano in condizioni di sfruttamento lavorativo sono in grado di organizzarsi e agire per modificare la propria situazione. A questo scopo, ci concentriamo sul fenomeno del cosiddetto caporalato agricolo, che è l'ambito in cui, più che altrove, si concentrano i fenomeni di sfruttamento e le possibilità di degenerazione in lavoro coatto o addirittura riduzione in schiavitù.

Mentre la prima parte dell'articolo è dedicata alla descrizione e alla storia del fenomeno del caporalato, nella seconda ci si concentra invece sulla descrizione di due studi di caso in due aree agricole della regione Puglia, nell'Italia meridionale: la provincia di Foggia e il comune di Nardò, in provincia di Lecce. La descrizione del primo caso si basa sulla ricerca di dottorato dell'autore, e fa ricorso, oltre che a dati statistici sul territorio e alla letteratura esistente sul caso, a informazioni raccolte tramite interviste semi-strutturate a sindacalisti, operatori del privato sociale, politici e impiegati pubblici che si occupano di immigrazione; per quanto riguarda Nardò, invece, gli eventi descritti in questo articolo sono stati osservati e analizzati da studiosi e attivisti sociali che si trovavano in loco quando essi sono avvenuti; i loro resoconti ${ }^{2}$ sono la fonte principale per la descrizione del caso, su cui si basa l'interpretazione qui fornita, presentata nelle conclusioni.

\section{Il caporalato}

Il caporalato è un fenomeno che nel dibattito politico italiano è collegato pressoché esclusivamente all'immigrazione nel Mezzogiorno, ma di fatto è una pratica che esiste in Italia, almeno dagli anni '70, e riguarda tutto il territorio nazionale, per quanto sia più forte e costante nelle regioni meridionali.

La definizione tecnica del caporalato è intermediazione illecita nell'avviamento al lavoro della manodopera agricola, proibita come tale dall'articolo 20 della legge 83/1970 ("Norme in materia di collocamento e accertamento dei lavoratori agricoli"), anche se, nella pratica, esso può applicarsi anche ad altri settori lavorativi, in particolare I'edilizia. I caporali mediano tra offerta e domanda di lavoro in una determinata area geografica e in un determinato settore, nel senso che si occupano di selezionare, al posto dei datori di lavoro, la manodopera necessaria alle attività, scegliendo tra gli aspiranti lavoratori. Questa processo di selezione (che si svolge in maniera informale, nell'ambito di un'economia in nero) non avviene una volta per tutte, ma anzi è solitamente giornaliero. 2 BRIGATE DI SOLIDARIETÀ ATTIVA, SACCHETTO, Devi, NIGRO, Gianluca, PERROTTA, Mimmo,
SAGNET, Yvan. Sulla pelle viva. Nardò: la lotta autorganizzata dei braccianti agricoli. 
Il funzionamento del compito di mediazione è il seguente: il caporale acquisisce le richieste di manodopera dei datori di lavoro, poi con mezzi propri raduna la quantità necessaria di braccianti e li porta sul luogo di lavoro per poi riportarli indietro alla fine della giornata lavorativa; mansioni e stipendi sono negoziati con i datori di lavoro direttamente dal caporale, il cui compenso consiste in una quota dei salari dei braccianti. Questo meccanismo sembra andare tutto a favore degli imprenditori, che trattengono i vantaggi dell'impiego di lavoro nero, e tutto a danno dei lavoratori, che perdono il vantaggio di poter usufruire del sistema di assistenza e devono corrispondere parte dei propri guadagni ai caporali. In realtà la distribuzione di costi e benefici è più sfumata, in questa fase, per alcuni motivi: innanzitutto, sino ad alcuni anni fa, e in parte anche oggi, i caporali appartenevano allo stesso contesto geografico, economico e sociale dei lavoratori - un lascito delle origini del fenomeno - quando i selettori di manodopera erano operai con stipendi poco superiori a quelle dei braccianti; I'appartenenza e la necessità di coltivare reti di relazioni, necessarie per svolgere bene il proprio compito, prevenivano i caporali dall'abusare della propria posizione di privilegio. A questo si aggiunga che la normalità dell'illegale ha facilitato e facilita molto l'accettazione del ruolo del caporale da parte dei lavoratori, che spesso vedono in questa figura una scelta preferibile rispetto alle trafile burocratiche dei canali ufficiali di accesso al lavoro. Dal canto loro, i datori di lavoro, pur godendo di innegabili vantaggi dalla presenza dei caporali, finiscono per diventare del tutto dipendenti da essi, poiché il caporalato tende alla monopolizzazione del mercato del lavoro informale. L'unica categoria che dalla situazione trae solo vantaggi è quindi proprio quella dei caporali, che diventano in punto di riferimento imprescindibile tanto per i lavoratori quanto per i datori di lavoro, e intorno alla propria centralità possono organizzare il lavoro in modo da massimizzare i propri benefici individuali ${ }^{3}$.

La caratteristica fondamentale del caporalato è che la sua opera di mediazione si concentra sulle fasce più deboli della forza lavoro: nei decenni passati era in particolare la manodopera femminile a trovare lavoro tramite i caporali, mentre a partire dagli anni '80 è aumentata sempre più la quota degli immigrati, provenienti in particolare, ma non solo, da Medio Oriente e Africa subsahariana. L'arrivo degli immigrati ha portato grandi cambiamenti anche nell'organizzazione del sistema, con l'emergere di caporali stranieri; se alcuni di essi hanno seguito il percorso del caporalato tradizionale (ovvero partire come semplici braccianti e diventare mediatori dopo aver ottenuto la fiducia dei datori di lavoro), la maggioranza segue invece un percorso nuovo, che solo in parte può essere assimilato a quello del caporalato classico.

\footnotetext{
3 CURCI, Sara. Nero invisibile normale. Lavoro migrante e caporalato in Capitanata, p. 65.
} 
I caporali stranieri non appartengono alle comunità agricole del territorio, né vi appartengono gli immigrati che vengono qui a lavorare; la necessità di mantenere buoni rapporti con i lavoratori oltre che con i datori di lavoro è dunque molto minore. Allo stesso tempo, i braccianti stranieri sono potenzialmente più difficili da controllare rispetto a quelli italiani, per via della loro maggiore predisposizione alla mobilità: non hanno vincoli familiari o economici con il territorio, e come stagionali non hanno particolari problemi a spostarsi verso zone che promettono salari o trattamenti migliori. Questi elementi, combinati insieme, aprono le porte all'uso della violenza da parte dei caporali come forma di controllo sui lavoratori stranieri, sino ad arrivare a forme di lavoro coatto.

La sopraccitata legge del 1970, abrogata nel 2008, prevedeva pene sia per i mediatori che per i datori di lavoro che ad essi si rivolgevano, ma in assenza di strumenti di controllo sul territorio non è stata sufficiente a indebolire la persistenza del fenomeno. Lo sfruttamento dei lavoratori stranieri in agricoltura è stato oggetto di ricerca e denunce da parte di sindacati e associazioni del terzo settore ${ }^{4}$, ed è stato, sin dalla prima fase dei flussi migratori verso I'Italia, uno dei principali fattori di conflittualità tra migranti coinvolti, datori di lavoro, comunità locali.

Nell'estate del 1989 a Villa Literno, un comune di provincia di Caserta dove si concentravano gli stranieri impiegati come braccianti nella raccolta del pomodoro, i tentativi dei migranti di opporsi alle richieste dei caporali e di ottenere il sostegno del sindacato avevano causato reazioni ostili nella comunità locale, e il 25 agosto uno di loro, Jerry Masslo, fu ucciso nel corso di una aggressione a fini di rapina. Questo omicidio ebbe un effetto simbolico importante e sui compagni di lavoro ${ }^{5}$ e sull'opinione pubblica italiana, favorendo la nascita di un movimento nazionale anti-razzista. Anche nei decenni successivi altri casi di conflitti e tensioni riguardanti gli immigrati sono stati legati alle loro condizioni di vita e lavoro nel settore agricolo dominato dal caporalato: I'esempio più noto è quello della cosiddetta rivolta di Rosarno, comune in provincia di Reggio di Calabria con un forte settore agricolo specializzato nella produzione di arance, dove nel 2010 le aggressioni ai danni di alcuni braccianti stranieri hanno portato a proteste dei migranti e tensioni tra loro e la cittadinanza durate parecchi giorni, che hanno ottenuto una forte visibilità nazionale ${ }^{6}$.

\footnotetext{
4 MEDICI SENZA FRONTIERE. I frutti dell'ipocrisia; IDEM. Una stagione all'inferno; GALOSSI, Emanuele. Immigrazione, sfruttamento e conflitto sociale. Una mappatura delle aree a rischio e quattro studi di caso territoriali; OSSERVATORIO PLACIDO RIZZOTTO (a cura di). Agromafie e caporalato: primo rapporto.

ANDALL, Jacqueline. New migrants, old conflicts: the recent immigration to Italy.

6 DEVITT, Camilla. La rivolta di Rosarno: gli immigrati si mobilitano?; MORRONE, Federica. Lo sfruttamento della manodopera straniera e le 'forme contemporanee di schiavitù': il caso di Rosarno; PUGLIESE, Enrico. Il lavoro agricolo immigrato nel Mezzogiorno e il caso di Rosarno.
} 


\section{Il caporalato in Puglia: tra scandali e mobilitazione dei migranti}

L'origine del caporalato in Puglia risiede nelle differenze di sviluppo tra le aree agricole della regione: a zone che, in seguito alla riforma fondiaria avviata nel 1950, avevano avviato processi di modernizzazione e coltura intensiva si contrapponevano zone rimaste arretrate, e con elevati tassi di disoccupazione. I braccianti senza lavoro di queste aree divennero fonte di manodopera per quelle più sviluppate, ma non si tradusse in flussi migratori intraregionali, bensì in pendolarismo, organizzato da alcuni lavoratori che, in virtù della loro esperienza e capacità, si assumevano il compito di selezione della manodopera per conto dei proprietari terrieri. Con gli anni il ruolo di tali mediatori divenne sempre più importante, per via delle crescenti difficoltà dei datori di lavoro a assumere autonomamente manodopera; al compito di selezione si affiancò quello del trasporto dei braccianti sul luogo di lavoro, la cosiddetta figura del caporale pullmanista, che corrisponde pressappoco a quella del caporale odierno ${ }^{7}$.

La dimensione del fenomeno varia da provincia a provincia, sulla base delle caratteristiche del settore agricolo nelle diverse aree. Nel caso della provincia di Foggia, parliamo di un territorio molto vasto, quello della piana del Tavoliere, che ha un'estensione di oltre 3000 chilometri quadrati, e dove l'agricoltura, che è l'attività predominante, è caratterizzata dalla prevalenza di aziende di piccole e medie dimensioni che utilizzano metodi di lavoro tecnologicamente poco sviluppati. Qui il lavoro agricolo, e in particolare la raccolta del pomodoro nei mesi estivi, è il più importante sbocco occupazionale per i lavoratori migranti, e infatti se il numero degli stranieri stabilmente residenti in provincia è molto basso, e nettamente inferiore alla media nazionale, le presenze durante i mesi estivi registrano invece un'impennata.

I migranti impegnati nel lavoro agricolo raramente alloggiano nei centri urbani, ma nelle cosiddette borgate, ovvero piccoli centri rurali nelle prossimità degli agri dove lavorano. Ci sono tre tipi di borghi: quelli abitati ormai solo da immigrati, borghi in cui gli immigrati convivono con gli autoctoni, e borghi a immigrazione invisibile, dove la popolazione immigrata si stabilisce in edifici dispersi intorno al borgo stesso, e non entrano a contatto con la popolazione locale $^{8}$. Questa situazione è legata alla non stanzialità dei migranti e alla periodicità dei flussi migratori: nelle borgate gli edifici utilizzati per dormire sono quasi sempre costruzioni abbandonate, come strutture agricole dismesse o chiese sconsacrate, le cui condizioni igienico-sanitarie sono precarie a dir poco; vi abita solo chi si ferma in Capitanata per periodi brevi, come i lavoratori stagionali. In contesti così precari, il caporalato ha buon gioco a imporsi,

\footnotetext{
ZACCARIA, Costanza. Lavoro nero oggi: il fenomeno del caporalato agricolo in Puglia, p. 72-73.

8 MINCUZZI, Simona. Le borgate della Capitanata, p. 214.
} 
poiché i lavoratori migranti non hanno altri referenti al di fuori dei caporali per accedere al lavoro, e proprio in provincia di Foggia possiamo osservare alcune delle degenerazioni coercitive del caporalato.

Nel 2006 è stata scoperta una rete di sfruttamento di immigrati polacchi, praticamente ridotti in schiavitù: salari bassissimi, inferiori a quelli promessi e decurtati dalle continue richieste di soldi per pagare l'alloggio, maltrattamenti da parte dei caporali, impossibilità di abbandonare il lavoro, casi di omicidio e di sparizioni. Lo scandalo ha messo in luce l'evoluzione del sistema del caporalato, e quella che potrebbe essere definita l'internazionalizzazione della figura del caporale pullmanista: i lavoratori polacchi vittime dei soprusi erano stati reclutati direttamente in Polonia, con la prospettiva di un lavoro faticoso ma pagato bene - rispetto agli stipendi locali - ed erano partiti su pullman dell'organizzazione di reclutamento, che li aveva portati in provincia di Foggia e subito affidati ai caporali/carcerieri, loro connazionali ${ }^{9}$.

Può sembrare strano che i più gravi episodi di maltrattamenti vedano come protagonisti immigrati neocomunitari, che in quanto tali godono di più tutele rispetto a extracomunitari e irregolari, ma in realtà questo risponde ad una logica ben precisa. Gli immigrati extracomunitari provenienti dall'Africa hanno un'esperienza decennale dei lavori in agricoltura, e anni di presenza in Italia hanno sviluppato un network di conoscenze e reti informative che permettono anche ai nuovi arrivati di orientarsi meglio in Italia e valutare dove andare a lavorare. In poche parole, rispetto ai più recenti immigrati est europei hanno un bagaglio informativo migliore; il che non vuol dire che non sono soggetti allo sfruttamento da parte dei caporali, ma che hanno un grado di autonomia che li tutela, sino a un certo punto, dalle organizzazioni che reclutano manodopera all'estero, le quali puntano sui soggetti più facilmente manipolabili: individui con poca o nulla esperienza migratoria, senza contatti e punti di riferimento nel paese di destinazione, senza progetti migratori di lungo termine. Un profilo più facile da ritrovare nei paesi di immigrazione recente, come appunto quelli dell'Europa orientale. Infine, va considerata la facilità di azione: trasportare lavoratori da un paese all'altro è più facile tra stati comunitari con libertà di movimento che non da un continente all'altro, soprattutto, con le restrittive norme sugli ingressi e la politica dei respingimenti attualmente in vigore.

In tutto questo, l'evoluzione del caporalato, da semplice sistema di mediazione a organizzazione con legami internazionali, è evidente; e ci si può chiedere se e quanto esso abbia legami con le organizzazioni criminali presenti sul territorio. È difficile dare una risposta precisa, ma è certo che nel corso degli anni Novanta l'interesse della criminalità organizzata in agricoltura

9 LEOGRANDE, Alessandro. Uomini e caporali. Viaggio tra i nuovi schiavi nelle campagne del Sud, p. 17-23. 
fosse rivolta più alla riscossione del pizzo sulla produzione di pomodoro, su cui si sono consumati anche scontri sanguinosi tra i clan ${ }^{10}$ che non al traffico della manodopera est europea. $\mathrm{Ci}$ sarebbe dunque un rapporto di convivenza pacifica tra caporalato e criminalità organizzata, più che contatti veri e propri. E comunque, è innegabile che nel caporalato siano riscontrabili elementi della mentalità mafiosa: già prima dell'arrivo degli immigrati, tra i diversi caporali sorgevano rivalità per il controllo della manodopera e i favori dei datori di lavoro; queste rivalità si mantengono su scala più ampia, tra i gruppi che reclutano manodopera straniera, organizzati su base nazionale. Per questo le statistiche sugli stranieri in Capitanata mostrano ondate periodiche di immigrati di diverse nazionalità: il gran numero di polacchi negli anni passati era legato all'azione di reclutamento in Polonia, ma dopo la scoperta dei casi di riduzione in schiavitù (che ha suscitato nel loro paese una reazione emotiva molto forte) e il successivo aumento dei controlli l'utilizzo di manodopera polacca ha subito una brusca frenata, per essere sostituita da quella romena (neocomunitaria dal 2007). II cambiamento della nazionalità prevalente non è stato accompagnato da un cambiamento delle condizioni di vita: le cronache del 2008 riportano notizie di maltrattamenti o scomparse, analoghe a quelle dello scandalo polacco del 2006. E, a fianco di casi estremi di riduzione in schiavitù, le ben più diffuse, pessime condizioni di vita dei lavoratori stranieri, alloggiati in edifici diroccati e privi di servizi igienici, con turni di lavoro estenuanti, in campi privi di punti per ritrovarsi o abbeverarsi. Questa situazione di degrado è stata denunciata in due rapporti di Medici senza frontiere, senza che ciò suscitasse reazioni particolari; ben più efficace è stato, nel 2007, il reportage del giornalista dell'Espresso Fabrizio Gatti ${ }^{11}$ che, fintosi immigrato, ha lavorato per un giorno nelle campagne del Tavoliere, documentando sia il meccanismo di reclutamento da parte dei caporali che le condizioni di vita e di lavoro dei braccianti; tuttavia, dopo una fase di maggiori controlli sull'onda dello scandalo, la situazione è tornata simile a quella precedente: pochi ispettori, verifiche sporadiche, e la legge regionale approvata dopo la scoperta dello sfruttamento dei polacchi e volta a contrastare il caporalato che non trova applicazione sul territorio.

Il caporalato ha un ruolo nelle precarie condizioni di vita degli immigrati: affidando completamente l'assunzione di manodopera a questi mediatori, i datori di lavoro si sentono deresponsabilizzati, e non si ritengono in dovere di fornire ai lavoratori servizi di alcun tipo. D'altra parte, nemmeno i caporali lo fanno, proprio per la sopraccitata inutilità di mantenere buoni rapporti con la manodopera: ciò che importa è solo il contenimento dei costi e la massimizzazione del profitto.

\footnotetext{
10 Ibidem, p. 224.

${ }^{11}$ GATTI, Fabrizio. Bilal. Viaggiare e morire da clandestini, p. 402-455.
} 
Quanto detto sinora sembra indicare che l'emergere di caporali stranieri abbia portato ad un aumento della violenza nel sistema agricolo del Foggiano, e a un peggioramento delle condizioni di vita dei lavoratori. Certo è così, ma si devono considerare alcuni elementi: innanzitutto, questa evoluzione violenta del caporalato è in linea con la filosofia del contenimento dei costi e della conflittualità sociale che è alla base della diffusione del fenomeno, non c'è una vera rottura con il passato; e, in tutti i casi, il caporalato in sé risponde ad una logica di sfruttamento e di violenza, simbolica e non, preesistente all'arrivo degli immigrati ${ }^{12}$. Si può anzi dire che la violenza fisica esplode proprio perché gli immigrati, a differenza degli autoctoni, non hanno interiorizzato l'ineluttabilità di questo sistema, e quindi alcuni di loro non sono disposti ad accettarlo senza ricorso alla coazione fisica.

A prescindere dalle ipotesi, quello che è certo è che la presenza dei caporali stranieri è utilizzata, a livello più o meno inconscio, dagli attori del sistema agricolo per giustificare la situazione precedente: si introduce una distinzione tra il caporalato classico, che in fondo non causa problemi, e il caporalato degli stranieri, violento e con tratti di disumanità ${ }^{13}$; la distinzione tra noi e loro legittima così le pratiche di mediazione illecita senza che questa legittimazione debba essere dichiarata esplicitamente: il caporalato autoctono non è più parte del problema, perché il problema viene attribuito in toto alla presenza immigrata. Eppure, è proprio questa percezione divisoria a rendere, nella realtà, ancora più sfocata la divisione tra caporalato classico e caporalato nuovo: i caporali locali traggono dalla presenza degli stranieri una ulteriore legittimazione che rafforza il loro ruolo agli occhi dei lavoratori; i quali lavoratori non superano le divisioni etniche sulla base della loro comune situazione, ma sono anzi divisi e in alcuni casi reciprocamente ostili: i braccianti italiani possono imputare agli immigrati la diminuzione dei salari (e i caporali italiani possono usare questa convinzione per giustificare trattamenti meno favorevoli), e i braccianti stranieri, di fronte all'ostilità dei colleghi locali, non hanno possibilità di integrarsi nelle comunità, restando quindi alla mercé dei caporali. Il circolo vizioso che ne deriva è estremamente difficile da spezzare, in assenza di una decisa azione politica: non è possibile, infatti, che sia il sistema economico e sociale da solo a produrre delle alternative, a causa della debolezza degli altri canali di accesso la lavoro: i centri di impiego sono depotenziati dalle numerose irregolarità già citate, e il sindacato, che pure spesso si impegna per imporre assunzioni regolari ai datori di lavoro, non gode di un seguito sufficiente per avere un impatto determinante, e anzi è stato accusato di aver contribuito allo sviluppo di pratiche clientelari, svolgendo un'opera propria di mediazione per

\footnotetext{
${ }^{12}$ CURCl, op. cit., p. 51-72.

13 Ibidem, p. 56.
} 
I'erogazione di risorse assistenziali. Il segretario della Flai-Cgil di Foggia sostiene che sia in aumento il numero di stranieri che si rivolgono al sindacato per aiuto, tutela e informazioni, ma la figura del lavoratore immigrato resta sempre quella di un soggetto più precario degli altri, più esposto ai maltrattamenti, più difficile da sindacalizzare; e infatti è sempre l'esponente sindacale a sottolineare l'importanza delle leggi per stroncare il fenomeno del caporalato, implicando così l'insufficienza di un'azione portata avanti solo dalle associazioni.

Gli effetti del caporalato sulle condizioni di vita dei lavoratori appaiono anche nello sviluppo di un altro fenomeno sociale, tipico della Capitanata, che è quello dei cosiddetti ghetti.

I ghetti sono, di solito, luoghi isolati lontani dai centri urbani; spesso nascono e crescono in borgate abbandonate, dove i migranti senza fissa dimora si stabiliscono e costruiscono case di fortuna senza acqua né altri servizi. Tra Rignano Garganico e San Severo si trova il più grande ghetto della provincia, che è stato spesso oggetto di attenzione giornalistica, ma ce ne sono molti altri più piccoli diffusi su tutto il territorio provinciale.

I ghetti non nascono dallo spontaneo concentramento di migranti in un unico luogo, o meglio: una volta che sono nati si sviluppano anche tramite la rete informale che spinge gli stranieri che arrivano in Capitanata per la prima volta negli stessi posti usati da chi li ha preceduti. Ma lo sviluppo originario dei ghetti, secondo uno dei miei intervistati, è stato funzionale al caporalato: nella sua forma originale, i caporali radunavano la forza lavoro nella principale piazza del paese e tra loro sceglievano i lavoratori giornalieri. Una dispersione eccessiva dei migranti sul territorio da un lato compromette la loro capacità di organizzarsi e dunque le loro richieste salariali, ma dall'altro rende più lungo e dispendioso il processo di selezione della forza lavoro. I ghetti, dove gli aspiranti lavoratori si concentrano, sono una soluzione.

Gli operatori di alcune associazioni che lavorano nei ghetti hanno detto nelle interviste che, oltre alle pessime condizioni igieniche, questi luoghi sono molto pericolosi perché favoriscono la diffusione di prostituzione e alcolismo. Ma al di fuori dei ghetti non ci sono alternative: le strutture esistenti non sono sufficienti e le amministrazioni locali di queste aree rurali sono spesso indifferenti al tema. Ciò causa la percezione - spesso non solo una percezione - dell'assenza delle istituzioni, un vuoto in cui i caporali offrono almeno un posto dove stare e un lavoro, non importa quanto faticoso o sottopagato.

Questa situazione comporta dei problemi per le organizzazioni del terzo settore che vogliono aiutare gli stagionali stranieri. Come ho descritto in altra sede $^{14}$, queste organizzazioni, che hanno un ruolo fondamentale nel fornire

${ }_{14}$ SCOTTO, Angelo. Focusing on the emergencies or on their roots? The role of nonprofit organisations in immigration policymaking in Italy, p. 58-62. 
prima accoglienza e assistenza ai migranti, sono divise su come comportarsi nei ghetti: da un lato l'obiettivo umanitario spinge a portare servizi e aiuto anche all'interno di essi, dall'altro vi è la consapevolezza che migliorare la qualità della vita all'interno dei ghetti rischia di legittimarli e di favorire quindi il rafforzamento del sistema del caporalato. Per cercare di superare questo problema alcune organizzazioni hanno promosso anche attività di formazione e empowerment per i migranti, per aiutarli a trovare altre possibilità lavorative, ma queste misure, chiaramente insufficienti di fronte ai numeri degli stagionali occupati in agricoltura, non bastano come soluzione, e la conseguenza è stata una divisione, all'interno del terzo settore, tra chi vuole continuare a portare servizi e assistenza nei ghetti e chi invece vuole puntare maggiormente sul contrasto legale del fenomeno del caporalato.

Nella descrizione del caso della provincia di Foggia abbiamo approfondito la descrizione del funzionamento del caporalato e dei suoi effetti e sulle condizioni di vita dei lavoratori migranti. Di fronte a questo fenomeno la politica è stata a lungo assente, attivandosi solo in seguito allo scandalo del 2006 e alle successive denunce giornalistiche, dopo le quali I'amministrazione regionale ha reagito sia promulgando una legge contro l'irregolarità nel lavoro agricolo sia creando delle strutture per ospitare i braccianti (i cosiddetti ‘alberghi diffusi'). Questa risposta, che ha avuto luogo dal 2007 in poi, non è stata l'unica reazione istituzionale al fenomeno: un'altra, stavolta anche a livello nazionale, si è avuta nel 2011 in seguito a quanto avvenuto a Nardò.

Nardò, comune in provincia di Lecce, ha anch'esso una tradizione agricola, basata sulle colture di angurie e pomodoro. Anche qui la raccolta del pomodoro attira lavoratori stranieri: a differenza che in Capitanata, però, a Nardò i migranti sono concentrati in un'unica area, quella della Masseria Boncuri, una struttura destinata proprio all'accoglienza dei braccianti stranieri e affidata dal comune di Nardò a due organizzazioni, le Brigate di solidarietà attiva e la cooperativa Finis Terrae. Anche a Nardò molti caporali sono stranieri, in particolare del Nord Africa, e si muovono liberamente nell'area di accoglienza della Masseria per reclutare i lavoratori.

II 30 luglio 2011 un gruppo di braccianti stranieri ha rifiutato la richiesta di un caporale di svolgere un'ulteriore mansione oltre quelle concordate per lo stesso salario. L'indisponibilità a soddisfare questa richiesta derivava anche dalla cattiva annata nella raccolta delle angurie, a causa della quale i braccianti avevano, sino a quel momento, lavorato e guadagnato poco $^{15}$. Ma in seguito a questo episodio i migranti della Masseria Boncuri hanno deciso di iniziare uno sciopero di protesta che ha compreso occupazioni delle strade, per impedire ai caporali di svolgere il proprio lavoro di raduno della manodopera, e sit-in di

${ }^{15}$ BRIGATE DI SOLIDARIETÀ ATTIVA et alii, op. cit., p. 150-151. 
fronte a sedi istituzionali per presentare le proprie richieste: aumenti salariali, abolizione del caporalato, creazione di vere liste di collocamento ${ }^{16}$. Lo sciopero non è stata una mossa estemporanea, ma una decisione presa collettivamente dai migranti riuniti in assemblea, che hanno anche eletto dei portavoce per rappresentarli nella trattativa con le istituzioni ${ }^{17}$. Questi portavoce sono stati anche i principali leader della protesta, e quelli che hanno cercato di portarla avanti nel momento in cui tra gli scioperanti sono emerse delle divisioni.

Se la richiesta di lavoro non pagato è stata la causa scatenante della protesta, essa è stata comunque l'ultimo episodio di una lunga serie di richieste eccessive e soprusi da parte dei caporali, che tutti insieme hanno creato un clima di malcontento nei lavoratori su cui si è innestata la protesta. Ad alimentare questo clima c'erano già state in zona, nei mesi precedenti, proteste e indagini contro episodi di sfruttamento dei migranti, non solo nel settore agricolo ${ }^{18}$, e quindi l'attenzione sul tema era più forte del solito, in particolare per gli enti gestori della Masseria Boncuri che hanno promosso nelle settimane precedenti la protesta una campagna di sensibilizzazione contro il lavoro nero ${ }^{19}$.

Lo sciopero ha visto all'inizio la partecipazione quasi compatta degli ospiti della Boncuri. Nelle due settimane successive il numero dei partecipanti è andato gradualmente diminuendo, per diversi motivi: le pressioni e minacce dei caporali, la necessità per i migranti di guadagnare, la disillusione per i pochi risultati ottenuti ${ }^{20}$. La protesta ha avuto sin da subito una notevole visibilità locale e nazionale, suscitando l'interessamento dei sindacati, dei partiti, delle amministrazioni locali e regionale. Due sono stati i principali risultati politici dello sciopero: un accordo tra alcune associazioni datoriali, i governi locali e il sindacato, stipulato I'8 agosto, in cui si istituivano liste di prenotazione per i lavoratori stagionali; e un decreto d'urgenza del governo del 13 agosto, che introduce il reato di intermediazione illecita e sfruttamento del lavoro ${ }^{21}$. A prescindere dall'efficacia di questo decreto, è chiaro che l'introduzione del reato non avrebbe avuto un effetto pratico immediato, e l'accordo sulle liste di prenotazione era stato considerato del tutto insufficiente dai migranti e dalle associazioni della Masseria, che pur partecipando alla riunione dell' 8 agosto non hanno ratificato gli accordi presi. Per questo gli scioperanti sono rimasti delusi dai risultati, e la delusione ha contribuito alla fine dello sciopero e alla divisione tra i migranti che sono tornati a lavorare e quelli che hanno cercato

\footnotetext{
16 Ibidem, p. 157.

17 Ibidem, p. 151.

18 Ibidem, p. 148-149.

19 Ibidem, p. 150.

20 Ibidem, p. 154-158.

21 Ibidem, p. 157-159.
} 
di continuare la protesta e di allargarla ad altre aree agricole interessate dal fenomeno del caporalato ${ }^{22}$.

Per quanto riguarda le reazioni dei caporali alla protesta, alcuni hanno cercato di calmare il malcontento pagando salari più alti e uguali per tutti, o saldando debiti pregressi; altri hanno invece cercato di piegare i manifestanti con minacce e avvertimenti. In generale, la protesta ha indebolito solo momentaneamente il caporalato, e in seguito all'esaurirsi dello sciopero e alla chiusura della Masseria Boncuri (la struttura è aperta solo per il periodo della raccolta) la situazione è tornata sostanzialmente quella di prima.

Lo sciopero dei migranti di Nardò è un esempio di movimento rivendicativo che è stato in grado di ottenere decisioni politiche dai detentori del potere. Ma se l'obiettivo concreto era riequilibrare i rapporti di forza tra braccianti e caporali, possiamo dire che esso non è stato raggiunto. Le cronache giornalistiche del 2015 hanno riportato casi di braccianti pugliesi, che lavoravano in regime di caporalato, morti nei campi a causa di colpi di calore e di orari di lavoro defatiganti; questi drammi hanno riportato all'attenzione dell'opinione pubblica il tema del caporalato; il che è anche prova che né il decreto governativo del 2011 né le leggi regionali hanno avuto successo nel contrastare il fenomeno del caporalato.

D'altra parte, dopo il 2011 non ci sono stati più casi simili di mobilitazione dei migranti, e questo non solo per via dei pochi risultati e dell'alto costo, in termini di ore di lavoro perse, della protesta: la stagionalità rende molto difficile, se non impossibile, la costituzione di un'organizzazione stabile, che non vada ricostruita da capo ogni anno; per raggiungere un simile obiettivo è probabilmente fondamentale il ruolo delle organizzazioni che offrono sostegno e servizi ai migranti, ma le attività degli enti che gestiscono la Boncuri si basano principalmente sul lavoro volontario, che è, anch'esso, una base troppo incerta per l'organizzazione duratura e stabile.

Inoltre, le notizie del 2015 aggiungono un ulteriore elemento al quadro: alcuni dei lavoratori morti nei campi erano italiani; infatti, anche se la percentuale di braccianti stranieri è molto aumentata dagli anni '80 in poi, essi non hanno mai soppiantato del tutto gli autoctoni. Ciò che qui conta è che i lavoratori italiani, nonostante non abbiano gli stessi limiti degli stagionali stranieri, non si sono mai mobilitati contro il caporalato, né lo hanno fatto dopo casi estremi come quelli degli ultimi mesi. Le motivazioni culturali, sociali e economiche che spiegano questa mancata reazione le abbiamo indicate nella descrizione del caporalato in provincia di Foggia, e si tratta di considerazioni che sono valide in pressoché tutte le aree in cui questo fenomeno ha una presenza storicamente consolidata.

22 Ibidem, p. 161. 


\section{Conclusioni}

La mobilitazione dei migranti stagionali può avere effetti positivi sull'integrazione degli stranieri in Italia? Nel caso specifico, la risposta è al momento negativa, perché la protesta contro il caporalato ha suscitato I'adesione solo di quei gruppi di cittadini italiani già interessati al tema dei diritti degli stranieri, ma non quella dei lavoratori in generale e di quelli agricoli in particolare. Diciamo "al momento" perché in via ipotetica la situazione potrebbe cambiare, ma qui entreremmo nel campo della pura speculazione.

Sulla base di quanto detto sinora, è evidente che lo sciopero di Nardò è stato un'eccezione e che la norma è l'assenza di reazioni collettive significative, come in Capitanata. Ma questo vuol dire che è preferibile, per le organizzazioni del terzo settore, non promuovere l'autorganizzazione di gruppo dei migranti? Tale dilemma si è posto, in forme più o meno esplicite, nel modo in cui le organizzazioni non profit della provincia di Foggia hanno cercato di affrontare la realtà dei ghetti, dividendosi sulle strategie migliori da seguire. La conseguenza di questa divisione è che ciò che manca in questa provincia non è tanto l'offerta di servizi e attività per aiutare i lavoratori stranieri, ma un impegno politico forte e coordinato contro il fenomeno del caporalato.

In estrema sintesi, in nessuna delle due province gli atteggiamenti di migranti e associazioni sembrano sufficienti ad avere un impatto significativo e nel miglioramento delle condizioni di vita dei braccianti e nel contrasto al caporalato. Inoltre, la mobilitazione dei migranti di Nardò risulta essere un episodio isolato reso possibile da un insieme di circostanze positive che non è detto si ripresentino di nuovo insieme in futuro. Tuttavia, se anche è stato solo un episodio, si tratta di un episodio che porta con sé osservazioni interessanti: e cioè che, in determinate condizioni, I'azione collettiva di migranti, anche se quasi del tutto estranei al tessuto socio-politico locale, può portare a effetti politici rilevanti. Proprio lo studio di queste condizioni può essere di grande interesse non solo per gli studiosi, ma anche per quei soggetti politici e sociali che si interessano di immigrazione.

\section{Riferimenti bibliografici}

ANDALL, Jacqueline. New migrants, old conflicts: the recent immigration to Italy. The Italianist, v. 10, n. 1, 1990, p. 151-174.

BRIGATE DI SOLIDARIETÀ ATTIVA; SACCHETTO, Davi; NIGRO, Gianluca; PERROTTA, Mimmo; SAGNET, Yvan. Sulla pelle viva. Nardò: la lotta autorganizzata dei braccianti agricoli. Roma: DeriveApprodi, 2012.

CURCI, Sara. Nero invisibile normale. Lavoro migrante e caporalato in Capitanata. Foggia: Edizioni del Rosone, 2008. 
DEVITT, Camilla. La rivolta di Rosarno: gli immigrati si mobilitano? In GUALMINI, Elisabetta; PASOTTI, Eleonora (a cura di). Politica in Italia. I fatti dell'anno e le interpretazioni. Edizione 2011. Bologna: il Mulino, 2011, p. 233-255.

GALOSSI, Emanuele. Immigrazione, sfruttamento e conflitto sociale. Una mappatura delle aree a rischio e quattro studi di caso territoriali. Roma: Istituto Ricerche Economiche e Sociali, 2011.

GATTI, Fabrizio. Bilal. Viaggiare e morire da clandestini. Milano: Rizzoli, 2007.

LEOGRANDE, Alessandro. Uomini e caporali. Viaggio tra $i$ nuovi schiavi nelle campagne del Sud. Milano: Mondadori, 2008.

MEDICI SENZA FRONTIERE. I frutti dell'ipocrisia. Roma: Sinnos, 2005.

MEDICI SENZA FRONTIERE. Una stagione all'inferno. Roma: Medici senza frontiere, 2008.

MINCUZZI, Simona. Le borgate della Capitanata. In RESTA, Patrizia (a cura di). Il vantaggio dell'immigrazione. Un progetto per una cultura condivisa. Roma: Armando, 2008.

MORRONE, Federica. Lo sfruttamento della manodopera straniera e le 'forme contemporanee di schiavitù': il caso di Rosarno. Diritti umani e diritto internazionale, v. 4, n. 2, 2010, p. 367-376.

OSSERVATORIO PLACIDO RIZZOTTO (a cura di). Agromafie e caporalato: primo rapporto. Roma: Ediesse, 2013.

PUGLIESE, Enrico. Il lavoro agricolo immigrato nel Mezzogiorno e il caso di Rosarno. Mondi Migranti, n. 3, p. 7-28, 2012.

SCOTTO, Angelo. Focusing on the emergencies or on their roots? The role of nonprofit organisations in immigration policymaking in Italy. Religion, State and Society, v. 44, n. 1, 2016, p. 51-64.

ZACCARIA, Costanza. Lavoro nero oggi: il fenomeno del caporalato agricolo in Puglia. La critica sociologica, n. 127, 1998, p. 72-86.

Articolo ricevuto il 25.07.2016

Accettato per la pubblicazione il 26.09.2016

Received for publication in July $25^{\text {th }}, 2016$

Accepted for publication in September 26 $6^{\text {th }}, 2016$

ISSN impresso 1980-8585

ISSN eletrônico 2237-9843

http://dx.doi.org/10.1590/1980-85852503880004806 